\title{
Prostatic Leiomyoma With Bizarre Nuclei: A Case Report
}

\author{
Vibeke M. Kristensen ${ }^{\mathrm{a}, \mathrm{c}}$, Anand C. Loya ${ }^{\mathrm{b}}$, Klaus Brasso ${ }^{\mathrm{a}}$
}

\begin{abstract}
Leiomyoma with bizarre nuclei (LM-BN) is a very rare finding which cannot be distinguished clinically from the very commonly occurring benign prostatic hyperplasia (BPH). If correctly diagnosed, LM-BN has a favorable prognosis after complete surgical excision. It is important to keep this alternative diagnosis in mind when treating a patient with complaints indicating prostatic hyperplasia. This case involves a 77-year-old male presenting with lower urinary tract symptoms; based on clinical presentation, the patient was diagnosed with BPH. A transurethral prostate resection was performed. Resected prostate tissue was collected for analysis and diagnosis of prostatic LM-BN was made.
\end{abstract}

Keywords: Prostate; Bizarre nuclei; Leiomyoma

\section{Introduction}

Leiomyoma is a benign smooth muscle tumor commonly found in the uterus, small bowel, and esophagus [1]. Smooth muscle elements associated with benign glandular hyperplasia of the prostate are frequent findings; however, pure prostatic leiomyoma - defined as an encapsulated mass of smooth muscle, $1 \mathrm{~cm}$ or more in diameter, devoid of glandular elements - is a rare finding [2].

Prostatic leiomyoma with bizarre nuclei (LM-BN) is even more seldom with only a handful of cases reported in the literature [1]. We report one case to heighten awareness of this entity. Prostatic LM-BN is a benign condition that when fully excised has a favorable outcome $[3,4]$.

\section{Case Report}

The patient, a 77-year-old male, was referred for medical exam-

\footnotetext{
Manuscript accepted for publication June 02, 2016

${ }^{a}$ Copenhagen Prostate Cancer Center, Department of Urology, Rigshospitalet, University of Copenhagen, Denmark

bDepartment of Pathology, Rigshospitalet, University of Copenhagen, Denmark

${ }^{\mathrm{c} C o r r e s p o n d i n g ~ A u t h o r: ~ V i b e k e ~ K r i s t e n s e n, ~ D e p a r t m e n t ~ o f ~ U r o l o g y, ~ R i g s h o s-~}$ pitalet, Blegdamsvej 3, Copenhagen 2100, Denmark.

Email: V.Kristensen@me.com
}

doi: http://dx.doi.org/10.14740/wjnu249w ination due to elevated liver enzymes. The patient complained of lower urinary tract disease symptoms. A physical examination revealed a benign enlargement of the prostate. The patient was referred to a urological clinic. No biopsies were taken prior to the patient having a transurethral resection of the prostate (TUR-P). The postoperative course was uneventful and the patient's voiding problems were resolved.

Primary histopathological examination based on prostatic tissue attained from the TUR-P procedure demonstrated prostatic leiomyoma. The patient was then referred for further urological evaluation at the Department of Urology, Rigshospitalet. At the first visit to our outpatient clinic, PSA was normal $(2.7 \mathrm{ng} / \mathrm{mL}$, ratio 0.36$)$. A transrectal ultrasonography showed an $80 \mathrm{cc}$ prostate with an isoechoic signal. Subsequent prostatic biopsies and revision of the primary specimen revealed a smooth muscle tumor with increased cellularity, large cells with frequent bizarre forms. Nuclei were pleomorphic and hyperchromatic and there were occasional typical mitotic figures (0-1/10 high power field). Scattered multinucleated tumor giant cells and few nuclei with pseudoinclusions were seen. There was no necrosis or hemorrhage (Figs. 1 and 2). The primary diagnosis was thus confirmed, and no prostatic malignancy was demonstrated. A CT scan showed no signs of metastasis. An MRI showed a $5 \times 6 \times 6 \mathrm{~cm}$ tumor of the prostate with apparent focal encasement of the right seminal vesicle, no enlarged lymph nodes or bony metastases were seen (Fig. 3). A PET-CT showed no signs of malignancy in the prostate. Liver enzymes normalized without intervention and no explanation of the previously elevated levels was found.

The patient has been followed conservatively; he is clini-

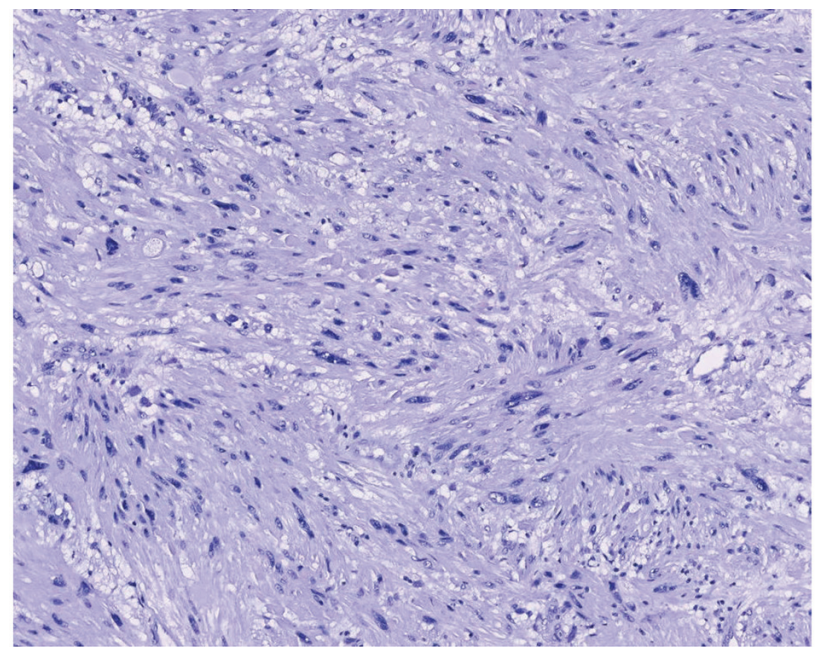

Figure 1. Prostate biopsy, $10 \mathrm{HPF}$, hematoxylin and eosin stain. 


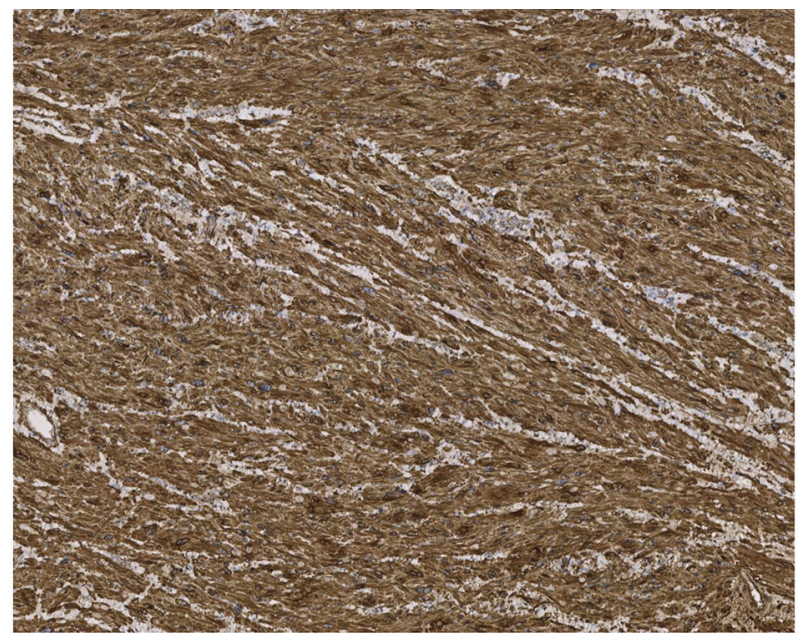

Figure 2. Prostate biopsy, smooth muscle antibody (SMA) stain.

cally stable without resurgence of voiding symptoms, and PSA has remained stable. During his course of treatment, the patient has reported repeated incidents of intermittent macroscopic hematuria. A diagnostic workup has found no alternative source of the bleeding. Urine analysis has revealed leukocytes and nitrate but no trace of blood. At this time, the patient has his next scheduled follow-up in 6 months. Treatment strategy is continued follow-up until obstructive voiding symptoms reappear, in the event of which a prostatectomy would be recommended.

\section{Discussion}

Prostatic leiomyoma was first reported in 1876 [4]. Prostatic leiomyoma was defined by Kaufman and Berneike in 1951 [2]. From the time of their definition less than one hundred case reports have been published, underlining the rarity of this condition [5]. Clinically prostatic leiomyoma mimics benign prostate hypertrophy (BPH). Prostatic leiomyomas are benign and in cases associated with voiding issues, surgical treatment often has long-standing effect. Recurrence does not occur where radical excision is carried out $[6,7]$.

LM-BN represents a subgroup of the leiomyomas, which has, to the best of our knowledge, been reported very rarely [8]. This LM-variant is being referred to as symplastic, pleomorphic or atypical leiomyoma; however, as per the 2003 WHO's classification for genital tumors, it has been designated "leiomyoma with bizarre nuclei" [9].

The origin of leiomyoma is not firmly established but some theorize that the neoplasm stems from repeated infections and inflammation, thereby transforming the glandular tissue to smooth muscle [3]. Others believe the neoplasm originates from embryonic Mullerian remnants [2].

LM-BN should be differentiated from stromal hyperplasia and stromal tumors of uncertain malignant potential (STUMP). STUMP can be distinguished by its histomorphology as well as its usually positive reaction for CD34 immunostaining and negative reactions for smooth muscle actin and desmin. Char-

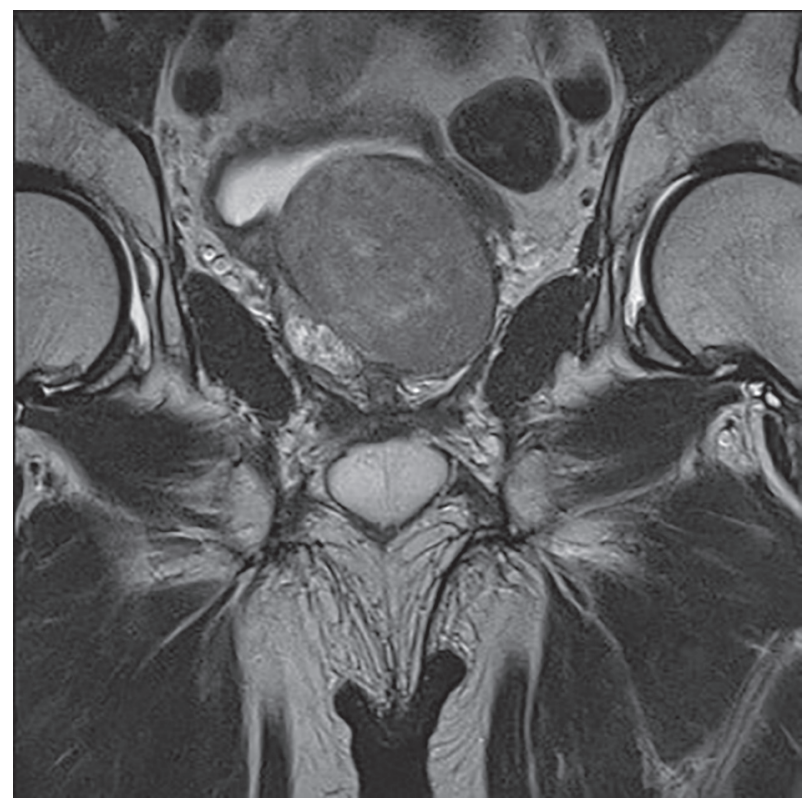

Figure 3. MRI, ventral view.

acteristic of LM-BN is the presence of pleomorphism, bizarre nuclear forms, and hyperchromatic nuclei. LM-BN can be differentiated from the relatively more frequent and aggressive leiomyosarcomas which exhibit necrosis and a dearth of mitosis. Histological examination is necessary to confirm the diagnosis [10]. However, it may be challenging to differentiate and categorize these specifically on prostate needle biopsies.

Rarity of LM-BN makes it difficult to differentiate from stromal hyperplasia, STUMPs, and leiomyosarcomas. It is important to remember LM-BN and that through careful observation, awareness of this rare leiomyoma variant can help the clinician arrive at a correct diagnosis and treatment.

\section{Conclusion}

Prostatic mesenchymal tumors are rare and cannot be distinguished from BPH on clinical examination. Often patients will present with an obstructive voiding pattern occasionally combined with hematuria. Once diagnosed, surgical treatment for voiding problems can be expected to have long-term effects.

\section{References}

1. Khalil KH, Rix GH, McBrien MP, al-Rufaie HK. Bizarre leiomyoma of the prostate. Br J Urol. 1997;79(4):660.

2. Kaufman JJ, Berneike RR. Leiomyoma of the prostate. J Urol. 1951;65(2):297-310.

3. Barba J, Tolosa E, Panizo AF, Berian JM. Prostatic leiomyoma. Case report. Arch Esp Urol. 2011;64(7):631-635.

4. van Ulden-Bleumink WM, Dom PG, Ramakers BP, van Adrichem NP. A rare prostatic diagnosis of an old man: a pure prostatic leiomyoma. Case Rep Urol. 2013;2013:741235. 
5. Tavora F, Kryvenko ON, Epstein JI. Mesenchymal tumours of the bladder and prostate: an update. Pathology. 2013;45(2):104-115.

6. Croce S, Young RH, Oliva E. Uterine leiomyomas with bizarre nuclei: a clinicopathologic study of 59 cases. Am J Surg Pathol. 2014;38(10):1330-1339.

7. Mellas S, Bouchikhi AA, Tazi MF, Khallouk A, Elammari JE, El Fassi MJ, Farih MH. Prostatic leiomyoma: a case report. Case Rep Urol. 2012;2012:702762.

8. Hossain D, Meiers I, Qian J, MacLennan GT, Bostwick
DG. Prostatic leiomyoma with atypia: follow-up study of 10 cases. Ann Diagn Pathol. 2008;12(5):328-332.

9. Hendrickson MR, Tavassoli F, Kempson RL, McCluggage WG, HallerU, Kubik-Huch RA. Mesenchymal tumors and related lesions. WHO Classification of Tumours Pathology and Genetics of Tumours of the Breast and Female Genital Organs. IARC Press. 2003:233-244.

10. Gupta R, Singh S, Khurana N. Leiomyoma of the prostate - a rare mesenchymal tumor: a case report. Indian J Pathol Microbiol. 2007;50(2):403-405. 